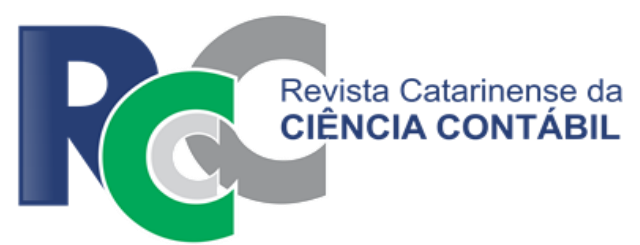

Revista Catarinense da Ciência Contábil, ISSN 1808-3781 - eISSN 2237-7662,

Florianópolis, SC, v. 16, n. 48, p.7-23, maio/ago. 2017

doi: $10.16930 / 2237-7662 /$ rccc.v16n48.2374 Disponível em http://revista.crcsc.org.br

\title{
APRENDIZADO AUTORREGULADO EM CONTABILIDADE: UMA ANÁLISE COMPARATIVA ENTRE DISCENTES DE MODALIDADE PRESENCIAL E A DISTÂNCIA*
}

\section{SELF-REGULATED LEARNING IN ACCOUNTING: A COMPARATIVE ANALYSIS BETWEEN CLASSROOM AND DISTANCE MODALITIES}

\author{
JOSÉ HILTON SANTOS AGUIAR \\ Mestrando em Contabilidade pela Universidade Federal da Bahia \\ (UFBA). Professor na Fundação Visconde de Caiu. Endereço: Rua do \\ Salete, 50 / Barris | 40070-200 / Salvador/BA / Brasil. \\ E-mail: hton87@gmail.com
}

\begin{abstract}
ANTONIO CARLOS RIBEIRO DA SILVA
Doutor em Desenvolvimento Curricular pela Universidade do MinhoPortugal. Professor no Programa de Pós-graduação da Universidade Federal da Bahia (UFBA). Endereço: Avenida Reitor Miguel Calmon, s/n / Vale do Canela / 40110-903 / Salvador/BA / Brasil.

E-mail: profacr@hotmail.com
\end{abstract}

\section{RESUMO}

Esta pesquisa realizou uma análise comparativa do perfil de estudantes de Ciências Contábeis da modalidade presencial e a distância quanto ao uso de estratégias autorreguladas na aprendizagem. Por meio de uma amostra de 302 estudantes de duas Instituições de Ensino Superior privadas, localizadas na Bahia, foi verificado como a modalidade de ensino, semestre, idade e gênero podem ser associados à utilização de estratégias de aprendizado autorregulado. Os dados foram tratados por meio de testes de médias (teste $t$ ), análises descritivas e análise fatorial. Os resultados apontaram que as estratégias mais empregadas pelos estudantes de contabilidade foram as de estabelecimento de objetivos e planejamento e a de memorização. Ao explicar as estratégias autorreguladas, por meio da modalidade de ensino, semestre, idade e gênero, os resultados indicaram que as médias são significativamente diferentes para a modalidade de ensino e idade. O estudo contribui para repensar os modelos de ensino-aprendizagem pautados no armazenamento de informações com o propósito de que exista fomento ao desenvolvimento de habilidades autônomas que promovam o aprendizado permanente fundamentado no ensinar a aprender ou aprender a aprender, características indicadas por órgãos internacionais e necessárias na excelência profissional.

Palavras-chave: Contabilidade. Autorregulação. Aprendizagem. Ensino em Contabilidade. 


\begin{abstract}
This research has conducted a comparative analysis of the profile of classroom and distance Accounting students as to the use of self-regulated strategies in learning. Through the sample of 302 students from two private higher-education institutions located in Bahia, it was verified how the mode of teaching, the semester, the age and the gender can be associated with the use of self-regulated learning strategies. Data has been treated by means of tests of averages ( $t$ test), descriptive analysis and factor analysis. The results showed that the strategies most employed by accounting students were the setting of goals, planning and learning. When explaining self-regulated strategies, through the teaching, semester, age and gender modes, the results indicate that the averages are significantly different for teaching and age modes. The study contributes in the sense of rethinking the teaching-learning models based on the storage of information aimed at encouraging the development of autonomous skills that promote the permanent learning based on the grounds of teaching for learning or learning how to learn, which are characteristics indicated by international bodies and necessary for professional excellence.
\end{abstract}

Keywords: Accounting. Self-regulation. Learning. Teaching in accounting.

\title{
1 INTRODUÇÃO
}

As relações de aprendizado foram historicamente estabelecidas pela presença síncrona entre os sujeitos da aprendizagem em espaço e tempo previamente definidos. O advento da educação superior paralelo à insuficiência da oferta de cursos de educação superior abriu espaço para o surgimento da educação a distância que se caracteriza pela separação entre professores e alunos de modo a requerer mais autonomia nos processos de aprendizagem (Gatti, 2001).

A autonomia é uma das particularidades apontadas pelos órgãos internacionais Accounting Education Change Comission [AECC] (1990) e American Institute of Certified Public Accountants [AICPA] (2000) como desejáveis no desenvolvimento de características permanentes na aprendizagem. De acordo com Chen e Paul (2003), na educação a distância o aprendiz é constructo do processo de aprendizagem, pois controla o ritmo dos estudos, determina a sequência das atividades.

Nessa perspectiva, Zimmerman e Martinez-Pons (1986) afirma que os indivíduos que controlam sua aprendizagem são autorregulados se selecionam métodos e estratégias de aprendizagem. Esses indivíduos ainda são capazes de estruturar o seu contexto de estudo e adaptam as suas estratégias de aprendizagem aos seus objetivos acadêmicos.

Diversos estudos têm apontado que as características do indivíduo afetam a aprendizagem (Chen \& Paul, 2003; Dias \& Leite, 2010; Cavanaugh, Lamkin, \& Hu, 2012; Bergamin, Ziska, Werlen, \& Siegenthaler, 2012) e que distintos comportamentos da autorregulação estão associados a diferentes resultados acadêmicos (Lynch \& Dembo, 2004). Outros estudos apontam existir diferenças significativas entre aluno autorregulado e outros que precisam de regulação externa no aprendizado (Arias, Barca Lozano, Gonzalez Cabanach, \& Núñez Pérez, 1999; Xu, Benson, Mudrey-Camino, \& Steiner, 2010; Richter \& Schmid, 2010). Indivíduos autorregulados são decididos, adotam estratégias, são persistentes e avaliam seus progressos; diferente daqueles que não traçam objetivos educacionais e consequentemente possuem dependência cognitiva, assim, pouca autorregulação na aprendizagem (Zimmerman, 2001).

Lima, Lima e Bruni (2015) verificaram as estratégias de aprendizado autorregulado de estudantes de contabilidade em duas universidades públicas baianas e associaram ao gênero, idade e semestre. Os resultados indicaram pouca apropriação das estratégias autorreguladas à medida que se avança o semestre em curso, fato que preocupou os autores, visto que é esperado que estudantes mais avançados no curso possuam maior apropriação de estratégias autorregulatórias. Thibodeaux, Deutsch, Kitsantas e Winsler (2016) analisaram a relação de uso de estratégias autorreguladas e o sucesso acadêmico à medida que 589 alunos avançavam nos cursos de psicologia e biologia em uma grande universidade americana. Os 
resultados apontaram que, no primeiro semestre, os alunos usavam gradativamente a estratégia de planejamento, pois destinavam mais tempo com a socialização, fato que refletia um menor desempenho acadêmico.

O objetivo desse estudo consiste em realizar uma análise comparativa do perfil de estudantes de Ciências Contábeis, da modalidade presencial e a distância, quanto ao uso de estratégias autorreguladas na aprendizagem. Para alcançar o objetivo geral, foram levantados os seguintes objetivos específicos: a) analisar as estratégias de autorregulação da aprendizagem adotada por discentes de contabilidade na modalidade presencial e a distância; b) relacionar a adoção das estratégias autorreguladas ao gênero, idade, semestre e modalidades de ensino presencial e a distância; c) verificar as grandezas associadas à modalidade de ensino, semestre, idade e gênero.

Este estudo visa a contribuir no desenvolvimento de uma revisão de literatura atualizada sobre autorregulação da aprendizagem e suas relações com estudantes de contabilidade. $O$ aprendizado em contabilidade necessita utilizar atributos que promovam a independência dos estudantes, ainda na graduação, de modo que posteriores alterações na mensuração, reconhecimento e divulgação das normas contábeis sejam aprendidas sem grandes dificuldades, e, desse modo, criar perspectivas para o preparo dos alunos para a vida profissional, principalmente nas atividades de aprender, manter habilidades e buscar o conhecimento (Schleifer \& Dull, 2009; Martin \& Dowson, 2009). Nesse contexto, torna-se imperioso ensinar os estudantes de contabilidade a aprender ou aprender a aprender, de modo a promover o desenvolvimento de habilidades autorregulatórias para um aprendizado permanente, recomendado por órgãos internacionais (AECC, 1990; AICPA, 2000) ao contador e desejável ao sucesso profissional.

\section{REFERENCIAL TEÓRICO}

\subsection{Autorregulação da aprendizagem}

De acordo Sternberg (2013), a psicologia cognitiva é a área de estudo que analisa como o indivíduo percebe, aprende e pensa as informações, de modo a compreender, por exemplo, porque determinados indivíduos se lembram de alguns fatos enquanto que outros não. Pesquisadores identificam duas abordagens que buscam o entendimento desses aspectos: a primeira acontece por meio de experiências internas que buscam a compreensão da natureza do racionalismo pela introspecção; e aquela que busca a compreensão a partir de estudos científicos das funções vitais por métodos empíricos, de modo que o racionalismo e o empirismo são o alicerce para a compreensão da mente humana (Sternberg, 2013).

Para Eysenck e Keane (1994), o desenvolvimento da comunicação fomentou as discussões sobre as teorias de sistemas de comunicação que posteriormente serviriam de base para o surgimento da psicologia cognitiva. Para Lefrançois (2008), a Psicologia Cognitiva analisa os processos mentais superiores, como a percepção, a memória, a formação de conceitos, linguagem, pensamento, solução de problemas e tomada de decisão de modo a inferir os processos mentais que se aprende como significado.

Nos anos de 1970 houve discussões no campo teórico da psicologia sobre aspectos que ligavam a relação entre a memória e o aprendizado, derivando o estudo da metacognição (Lima \& Bruni, 2012). Dessa forma, a metacognição foi definida como o domínio que o indivíduo possui sobre seu próprio conhecimento; e ainda na mesma década é compreendida como o domínio dos processos e produtos cognitivos (Flavell, 1976). Entre diversas definições sobre a metacognição, existe uma similaridade que aborda uma característica comum de mecanismos, a qual resulta no controle e autorregulação do processo intelectual.

Ao compreender que a partir da metacognição é possível a autorregulação intelectual, foram iniciadas pesquisas lideradas por Zimmerman para o entendimento da autorregulação da aprendizagem ou Self Regulated Learn (SLR). Com influências de paradigmas construtivistas, que tem o indivíduo como agente de seu aprendizado (Arias et al., 1999; Xu et al.,2010; Richter \& Schmid, 2010), Zimmerman (2001) aborda que os indivíduos são considerados autorregulados quando são persistentes, decididos, estratégicos e capazes de avaliar seus 
progressos; aqueles que são mais dependentes cognitivamente são, portanto, pouco autorregulados. Para Ribeiro (2003), a realização de autocrítica, a reflexão pessoal, a capacidade de realização da autocrítica, a modificação de hábitos de estudos são características de estratégias metacognitivas.

Para Simons e Beukhof (como citado em Figueira, 1994), a autorregulação é a habilidade de o indivíduo ser 'auto-ensinante', capaz de preparar, facilitar e regular a aprendizagem de forma a gerar um feedback e julgamento sobre o processo. Segundo Costa (2001), a autorregulação é caracterizada pelo grau de envolvimento ativo no processo de aprendizagem (metacognição, motivação e comportamental); comportamento de mudança cíclico (controle da eficácia, envolvimento e reflexão dos resultados); e dependência dos aspectos motivacionais (grau de envolvimento com relação de controles e crenças).

\subsection{Modelo de autorregulação da aprendizagem proposto por Zimmerman}

O modelo de autorregulação proposto por Zimmerman (2000) é dividido em fases, componentes e processos que produzem resultados na aprendizagem. A primeira fase aborda a antecipação/preparação e estabelece os objetivos e planos para alcançar as metas escolhidas e ocorre com a influência de aspectos motivacionais (autoeficácia), o objetivo é de valorização da aprendizagem. Na segunda fase há a execução e o controle que tem como propósito cumprir os objetivos traçados na primeira etapa. Há a necessidade da automonitoração por meio do uso de estratégias de aprendizado e do controle da atenção. A última etapa acontece com a autorreflexão e autorreação que envolvem o julgamento, autoavaliação e atribuições de causas sobre os objetivos estabelecidos na primeira fase e resultam em satisfação ou insatisfação, presença de reações (autorreflexão) e defensivas, com resistência e abandono ou satisfação e valorização pessoal. Essas três fases correspondem a um processo cíclico que através da retroalimentação permite alterações e contínuos aprimoramentos (Polydoro \& Azzi, 2009; Zimmerman, 2000).

Com a aprendizagem autorregulada se impulsiona a autonomia, em que a identificação dos erros é essencial para agregar novos conhecimentos, de modo a conduzir de forma prazerosa aos acertos e ao crescimento de novas aprendizagens. Para Jones, Alexander e Estell (2010), é esperado do aluno que possua comportamentos autorregulatórios, motivação e capacidade regulação do aprendizado para que seja possível automonitorar e autogerir o seu aprendizado. A aprendizagem autorregulada foi fundamentada na Psicologia e na Sociologia e Korkmaz \& Kaya (2012) apresentam um contexto em que alunos definem as tarefas, ajustam objetivos, criam planos, usam ferramentas, táticas e estratégias para execução de suas atividades. Para Zimmerman (2000), o desenvolvimento de estratégias de autorregulação por docentes é fundamental na promoção do aprendizado autorregulado (Zimmerman, 2000) e podem ser passadas aos alunos ao longo do curso. É integrada como treino autorregulatório (Zimmerman, Bonner, \& Kovach, 1986).

Zimmerman e Martinez-Pons (1986) desenvolveram 14 estratégias do aprendizado autorregulado. Segundo esses autores, o uso dessas estratégias confere ao aluno um valioso ferramental. Sua utilização é altamente correlacionada com os índices de sucesso acadêmico. As estratégias são: a) Autoavaliação; b) Organização e transformação; c) Estabelecimento de objetivos e planejamento; d) Procura de informação; e) Apontamentos; f) Estrutura Ambiental; g) Autoconsequências; h) Repetição e memorização; i) Ajuda de professores; j) Ajuda de pares próximos; k) Ajuda de especialistas; I) Revisão das anotações; m) Revisão de testes; e n) Revisão da bibliografia.

Ao comparar o nível de aprendizado autorregulado entre a modalidade presencial e a distância na educação superior, Sizoo, Malhotra e Bearson (2003) não encontraram diferenças significativas entre as duas modalidades, exceto na modalidade a distância quando estudantes do sexo feminino apresentaram maiores frequências na apropriação de estratégias autorreguladas do que na presencial.

Barnard-Brak, Paton e Lan (2010) realizaram um estudo na educação superior, na modalidade a distância, e identificaram a presença de cinco perfis de aprendizado autorregulado que variam de superautorregulados a nenhuma ou pouca autorregulação. Os resultados apontaram que os cinco perfis influenciam diretamente no desempenho acadêmico 
dos entrevistados, de modo que aqueles que possuem pouca ou nenhuma autorregulação apresentam desempenhos acadêmicos mais baixos do que aqueles que tendem a ser superautorregulados.

De modo geral, há consenso entre diversos pesquisadores de que a capacidade de autorregulação dos discentes é essencial na melhor qualidade da aprendizagem, desempenho, tomada de decisão, resolução de problemas e utilização do tempo (Schunk, 2001; Zimmerman, 2002; Boruchovitch, 2004; Dias \& Leite, 2010; Frison \& Moraes, 2010; Rosário, Nunes, Magalhães, Rodrigues, Pinto, \& Ferreira, 2010; Bergamin et al., 2012; Lima \& Bruni, 2012; Simão; Frison, 2013).

Nesse ínterim, com o propósito de relacionar o semestre em curso e as estratégias de autorregulação, Thibodeaux et al. (2016) analisaram a associação de uso de estratégias autorreguladas e o sucesso acadêmico à medida que 589 alunos avançavam nos cursos de psicologia e biologia em uma grande universidade americana. Os resultados apontaram que, no primeiro semestre, os alunos usavam gradativamente a estratégia de planejamento pois destinavam mais tempo com a socialização, fato que refletia um menor desempenho acadêmico.

Pesquisas que envolveram a idade do indivíduo com a autorregulação da aprendizagem foram desenvolvidos por Castel, Murayama, Friedman, McGillivray e Link (2013). Os autores analisaram como jovens e adultos utilizam estratégias de aprendizagem metacognitiva e as relacionou com o tempo de estudo. O resultado apontou que os adultos possuem maior controle autorregulatório nas estratégias de estudo e na organização do tempo. Já pesquisas que envolveram autorregulação e gênero foram sondadas por Bembenutty (2007). Esse último autor investigou que as estratégias de autorregulação e o desempenho acadêmico variam em função dos gêneros e das etnias em um curso universitário. Os resultados apontaram que a nota final do curso e as estratégias de autorregulação não apresentaram diferenças quando observados a etnia e o gênero.

Castro (2016) analisou características da autorregulação da aprendizagem no ensino superior na modalidade a distância. Os resultados apontaram que os estudantes da modalidade analisada desenvolveram habilidades e atitudes típicas da autorregulação da aprendizagem que visam a maximizar suas aprendizagens em processos educacionais. Lima et al. (2015) analisaram as estratégias de aprendizados propostas por Zimmerman e MartinezPons (1986) e relacionaram-nas ao gênero, idade e semestre em curso em 249 estudantes da modalidade presencial de duas universidades de ensino presencial na Bahia. Verificaram que o gênero e a idade são fatores que influenciam o grau de autorregulação de um aluno. Mulheres e discentes mais jovens tendem apresentar melhores níveis de aprendizagem autorregulada. Entretanto, nada foi possível ser constatado quanto à relação entre semestre e apropriação de estratégias autorreguladas ao longo do curso.

\section{PROCEDIMENTOS METODOLÓGICOS}

A metodologia utilizada foi a hipotético-dedutivo e exploratória, uma vez que se busca maior familiaridade com o fenômeno (Gil, 1991) entre as possíveis relações da modalidade presencial e à distância quanto à autorregulação da aprendizagem.

O universo correspondeu a aproximadamente 1000 alunos matriculados no primeiro semestre de 2015 na Fundação Visconde de Cairu (FVC), localizada no município de Salvador (BA) e Universidade Norte do Paraná (UNOPAR), no polo do município de Feira de Santana (BA). A coleta de dados aconteceu por meio de questionários impressos dispostos em dois blocos: o primeiro indagava sobre as características pessoais do entrevistado e o segundo, num intervalo escalar de resposta que variou de 1 (nunca) e 7 (sempre), averiguou-se as reações dos estudantes em situações de aprendizado à luz das estratégias identificadas por Zimmerman e Martinez-Pons (1986) sobre o aprendizado autorregulado (Tabela 1). 
Tabela 1

\section{Estratégias de aprendizagem autorreguladas identificadas por Zimmerman e Martinez- Pons (1986) e proposições equivalentes indagadas}

\begin{tabular}{|c|c|}
\hline $\begin{array}{l}\text { Estratégias autorreguladas (Zimmerman } \\
\text { e Martinez-Pons, 1986) }\end{array}$ & Proposições indagadas \\
\hline 1. Autoavaliação & $\begin{array}{l}\text { 1. Após conclusão de um trabalho, sempre verifico para ter a } \\
\text { certeza que está bom. }\end{array}$ \\
\hline 2. Organização e transformação & $\begin{array}{l}\text { 2. Procuro sempre elaborar um plano (esquema) antes de } \\
\text { iniciar um trabalho. }\end{array}$ \\
\hline $\begin{array}{l}\text { 3. Estabelecimento de objetivos e } \\
\text { planejamento }\end{array}$ & $\begin{array}{l}\text { 3. Se tenho prova, começo a estudar o mais cedo possível, } \\
\text { para ficar descansado e tranquilo no dia. }\end{array}$ \\
\hline 4. Procura de informação & $\begin{array}{l}\text { 4. Antes de iniciar um trabalho, recorro sempre à biblioteca (e } \\
\text { outros meios de pesquisa seja físico ou digital) para separar o } \\
\text { máximo de informação sobre o tema. }\end{array}$ \\
\hline 5. Tomada de apontamentos & $\begin{array}{l}\text { 5. Sempre procuro anotar o máximo de apontamento de um texto } \\
\text { lido ou da aula expositiva do professor. }\end{array}$ \\
\hline 6. Estrutura ambiental & $\begin{array}{l}\text { 6. Para ter melhor concentração, procuro sempre ambiente que não } \\
\text { proporcione distração. }\end{array}$ \\
\hline 7. Autoconsequências & $\begin{array}{l}\text { 7. Quando faço uma prova, se ocorrer bem, ofereço-me } \\
\text { recompensa; caso ocorra o contrário, abro mão de algo que tanto } \\
\text { queria. }\end{array}$ \\
\hline 8. Repetição e memorização & $\begin{array}{l}\text { 8. Utilizo estratégias para memorizar o assunto (ou fórmulas) até } \\
\text { saber de cor o assunto a ser estudado. }\end{array}$ \\
\hline $\begin{array}{l}\text { 9. Ajuda de professores; } 10 \text {. Ajuda de } \\
\text { pares próximos; } 11 \text {. Ajuda de especialistas }\end{array}$ & $\begin{array}{l}\text { 9. Quando surge dificuldade e não consigo resolver sozinho, busco } \\
\text { ajuda externa (professor, colegas, outros). }\end{array}$ \\
\hline $\begin{array}{l}\text { 12. Revisão das anotações; } 13 \text {. Revisão } \\
\text { de testes e } 14 \text {. Revisão da bibliografia }\end{array}$ & $\begin{array}{l}\text { 10. Avalio o meu desempenho, vejo o que devo melhorar, visando a } \\
\text { preparar-me para uma prova. }\end{array}$ \\
\hline
\end{tabular}

Nota. Fonte: Adaptado de Lima, R. N., Filho, \& Bruni, A. L. (2012). Aprendizado Autorregulado em Contabilidade: Diagnóstico, Dimensões e Explicações. Anais do Encontro da Anpad (EnAnpad), Rio de Janeiro, RJ, Brasil, 36.;

Zimmerman, B. J., \& Martinez-Pons, M. (1986). Development of a structured interview for assessing student use of self-regulated learning strategies. American Educational Research Journal, 23, 614-628.

A amostra correspondeu a 335 estudantes presentes na sala de aula na primeira quinzena de julho de 2015, inclusive os estudantes da modalidade de ensino a distância. Foram descartados 33 questionários não respondidos integralmente, resultando na análise de 302 casos.

Para realizar a análise dos dados, foram utilizados três procedimentos quantitativos no intuito de alcançar os objetivos específicos: análise descritiva, para identificar as estratégias de aprendizagem autorreguladas; o teste paramétrico de médias (teste $t$ ) para analisar como as estratégias podem ser explicadas a partir da modalidade de ensino, estágio (semestre), idade e gênero; e a análise fatorial para verificar as grandezas associadas à modalidade de ensino. No teste paramétrico de médias, a amostra foi dividida em dois grupos: o gênero (masculino e feminino), o semestre em curso (até o quinto semestre e a partir do sexto semestre), a modalidade do curso (presencial ou EAD) e a idade (até 24 anos e acima de 24 anos).

Frisa-se que pesquisas anteriores utilizaram o mesmo critério para análise da segregação do semestre (Lima et al. 2015), da idade (Mayville, 2007; Lima et al. 2015), do gênero (Bembenutty, 2007; Lima et al. 2015) e da modalidade de ensino (Castro, Miranda, \& Leal, 2015).

As hipóteses que nortearam o alcance do objetivo específico (b) que busca relacionar a adoção das estratégias autorreguladas ao gênero, idade, semestre e modalidades de ensino presencial e à distância, assim como os respaldos teóricos (conforme o referencial teórico) que sustentam as hipóteses, são demonstradas na Tabela 2. 
Tabela 2

\begin{tabular}{ll} 
Hipóteses associadas aos objetivos do estudos e fundamentações teóricas \\
\hline Hipóteses & Fundamentação \\
\hline $\begin{array}{l}\text { H1 - O sexo feminino apresenta maior apropriação de } \\
\text { estratégias de autorregulação de aprendizagem. }\end{array}$ & $\begin{array}{l}\text { Pavesi (2015); Lima e Bruni (2012); } \\
\text { Bembenutty (2007) }\end{array}$ \\
\hline $\begin{array}{l}\text { H2 - A modalidade a distância se apropria de estratégias } \\
\text { autorreguladas de forma semelhante aos da modalidade } \\
\text { presencial }\end{array}$ & Sizoo et al. (2003) \\
\hline $\begin{array}{l}\text { H3 - A idade influencia a apropriação de estratégias de } \\
\text { aprendizagem autorregulada }\end{array}$ & Lima e Bruni (2012) Mayville (2007) \\
\hline $\begin{array}{l}\text { H4 - Não há relação entre maior utilização de estratégias e o } \\
\text { semestre em curso. }\end{array}$ & Lima e Bruni (2012) \\
\hline
\end{tabular}

Nota. Fonte: Elaborado pelos autores (2017).

\section{RESULTADOS E ANÁLISES}

Esta pesquisa realizou uma análise comparativa do perfil de 302 estudantes de Ciências Contábeis, da modalidade presencial e a distância, quanto ao uso de estratégias autorreguladas na aprendizagem em duas IES privadas localizadas na Bahia. A característica da amostra analisada pode ser visualizada na Tabela 3.

Tabela 3

Características da amostra estudada

\begin{tabular}{|c|c|c|c|c|c|c|c|c|c|}
\hline Gênero & & Isculino & & eminino & & & & & Soma \\
\hline & Presencial & EAD & Presencial & EAD & & & & & \\
\hline $\mathrm{Fi}$ & 41 & 77 & 90 & 94 & & & & & 302 \\
\hline $\mathrm{Fi} \%$ & 13,58 & 25,50 & 29,80 & 31,13 & & & & & 100,00 \\
\hline Idade & até 20 & 21 a 25 & 26 a 30 & 31 a 35 & $>35$ & & & & \\
\hline EAD (Fi) & 12 & 29 & 56 & 37 & 37 & & & & 171 \\
\hline $\mathrm{Fi} \%$ & 9,60 & 9,60 & 18,54 & 12,25 & 12,25 & & & & 56,62 \\
\hline Presencial(Fi) & 10 & 33 & 32 & 27 & 29 & & & & 131 \\
\hline $\mathrm{Fi} \%$ & 3,31 & 10,93 & 10,60 & 8,94 & 9,60 & & & & 43,38 \\
\hline Semestre & 1 & 2 & 3 & 4 & 5 & 6 & 7 & 8 & Soma \\
\hline EAD (Fi) & 33 & 19 & 26 & 23 & 26 & 12 & 12 & 20 & 171 \\
\hline $\mathrm{Fi} \%$ & 10,93 & 6,29 & 8,61 & 7,62 & 8,61 & 3,97 & 3,97 & 6,62 & 56,62 \\
\hline Presencial(Fi) & 37 & 0 & 0 & 0 & 30 & 6 & 44 & 14 & 131 \\
\hline $\mathrm{Fi} \%$ & 12,25 & 0,00 & 0,00 & 0,00 & 9,93 & 1,99 & 14,57 & 4,64 & 43,38 \\
\hline Soma & 70 & 19 & 26 & 23 & 56 & 18 & 56 & 34 & 302 \\
\hline Soma Fi\% & 23,18 & 6,29 & 8,61 & 7,62 & 18,54 & 5,96 & 18,54 & 11,26 & 100,00 \\
\hline
\end{tabular}

Nota. Fonte: Dados da pesquisa (2017).

A Tabela 3 expõe que mais de $60 \%$ (Frequência simples- $\mathrm{Fi}$ ) dos graduandos em contabilidade são do sexo feminino, o que converge e ratifica os achados de Lima et al. (2015) sobre a representatividade do sexo feminino nos cursos de Ciências Contábeis nos cursos presenciais. A maior participação feminina em cursos de contabilidade é um achado importante, pois promove a diversidade, sobretudo, ao explorar as habilidades nas diversas áreas de atuação contábil. Entretanto, também é preciso fomentar a participação das mulheres na produção científica e na participação de eventos em contabilidade, pois, segundo Luca, Gomes, Corrêa e Domingos (2011), a participação feminina é relativamente baixa em publicação de eventos científicos, quando comparado ao gênero masculino, principalmente em eventos da USP e do ANPAD.

Quando é analisada a idade da amostra, verifica-se não haver grandes disparidades entre as modalidades de ensino presencial ou EAD. Entretanto, os dados apontaram que mais de $70 \%$ dos analisados possuem idade superior a 26 anos, diferentemente dos achados de Lima et al. (2015) em duas instituições públicas na Bahia, em que $73 \%$ da amostra possuía idade de até 25 anos. Tal comparação leva a inferir que o tipo de IES (pública ou privada) e a 
modalidade de ensino atraem públicos distintos em cursos de contabilidade no Estado baiano. Com esse raciocínio, estudantes com idade até 25 anos procuram por cursos em Universidades públicas enquanto aqueles com mais de 26 anos optam por instituições privadas e/ou o ensino a distância. Tais escolhas podem ser explicadas pela necessidade empregatícia e/ou pela constituição familiar, por exemplo.

A análise da Tabela 3 também elucida que a amostra é composta por $56,62 \%$ de estudantes da Universidade Norte do Paraná (UNOPAR), que cursam Ciências Contábeis na modalidade à distância e $43,38 \%$ de discentes que frequentam o mesmo curso na modalidade presencial na Fundação Visconde de Cairu, ambos no período noturno. O crescimento da EAD no Brasil tem sido inegável, porém a qualidade do ensino gera dúvidas e temores. Nesse interim, Nascimento e Junqueira (2011) verificaram se existe diferença significativa no desempenho da disciplina Contabilidade Introdutória entre os estudantes das modalidades a distância e presencial e concluíram que não existem tais diferenças, pois as médias finais analisadas não foram significativamente diferentes para as duas modalidades.

O primeiro objetivo específico deste estudo propõe analisar as estratégias de autorregulação da aprendizagem adotadas por discentes de contabilidade na modalidade presencial e a distância em duas Instituições Privadas que oferecem o curso de Ciências Contábeis na Bahia: Fundação Visconde de Cairu (FVC) e Universidade Norte do Paraná (UNOPAR). Os resultados estão apresentados na Tabela 4, dispostos para ambas as modalidades.

A Tabela 4 apresenta que a estratégia 1 (E1) é aquela mais adotada por estudantes da modalidade de ensino presencial $(90,1 \%)$ e a distância $(88,3 \%)$. De modo que, estudantes de contabilidade de ambas as modalidades tendem a realizar a autoavaliação com frequência quando finalizam/realizam alguma atividade. A maior adoção dessa estratégia corrobora uma parte da etapa três abordada por Zimmerman (2000) e Polydoro e Azzi (2009), que consiste na autorreflexão e autorreação que envolvem o julgamento, a avaliação e a análise de objetivos estabelecidos, que resultam em satisfação ou insatisfação. Para Melchior (1994), a autoavaliação é a capacidade de analisar os esforços gastos em relação as suas capacidades e os resultados obtidos ao que foi solicitado. Contribui para melhorar o desenvolvimento da autoavaliação ou em outras situações. Desse modo, a utilização dessa estratégia por estudantes de contabilidade confere um valioso ferramental para o desenvolvimento de senso crítico sobre os saberes aprendidos ao longo do curso, estratégia essencial no exercício da profissão para a elaboração e conferência de dados e demonstrativos contábeis.

\section{Tabela 4}

\section{Utilização de estratégias autorreguladas com adoção de um ponto médio}

\begin{tabular}{c|l|ccccccccccc}
\hline $\begin{array}{c}\text { Tipo de } \\
\text { modalidade }\end{array}$ & \multirow{2}{*}{ Ponto médio } & \multicolumn{10}{c}{ Estratégias (\%) } \\
\cline { 3 - 12 } & $\mathbf{1}$ & $\mathbf{2}$ & $\mathbf{3}$ & $\mathbf{4}$ & $\mathbf{5}$ & $\mathbf{6}$ & $\mathbf{7}$ & $\mathbf{8}$ & $\mathbf{9}$ & $\mathbf{1 0}$ \\
\hline \multirow{3}{*}{ Distância } & menor que 4 & 11,7 & 18,7 & 28,1 & 19,3 & 25,1 & 14 & 71,9 & 37,4 & 13,5 & 5,8 \\
& maior que 4 & 88,3 & 81,3 & 71,9 & 80,7 & 74,9 & 86 & 28,1 & 62,6 & 86,5 & 94,2 \\
& Total & 100,0 & 100,0 & 100,0 & 100,0 & 100,0 & 100,0 & 100,0 & 100,0 & 100,0 & 100,0 \\
\hline \multirow{3}{*}{ Presencial } & menor que 4 & 9,9 & 30,5 & 55 & 21,4 & 20,6 & 15,3 & 66,4 & 38,2 & 8,4 & 17,6 \\
& maior que 4 & 90,1 & 69,5 & 45 & 78,6 & 79,4 & 84,7 & 33,6 & 61,8 & 91,6 & 82,4 \\
& Total & 100,0 & 100,0 & 100,0 & 100,0 & 100,0 & 100,0 & 100,0 & 100,0 & 100,0 & 100,0 \\
\hline
\end{tabular}

Nota. Fonte: Dados da pesquisa (2017).

O ponto médio do instrumento de coleta de dados utilizado nesta pesquisa correspondeu a média 4 (quatro), conforme a Tabela 4, como ponto de segmentação entre maior ou menor apropriação das estratégias analisas. Percebe-se, ainda na Tabela 6 , que na modalidade a distância todas as estratégias são utilizadas com índice superior a $60 \%$, com exceção da estratégia 7 (E7) "autoconsequências", de modo que não é utilizada a "autopremiação" e/ou "autopunição" pela ocorrência de sucesso ou insucessos pelo desempenho de uma atividade. Essa estratégia (E7) é aquela menos utilizada por estudantes na modalidade presencial, assim como também houve pouca indicação do uso da estratégia 3 (E3) "estabelecimento de objetivos e planejamento" (45\%), o que indica que a maior parte dos 
estudantes da modalidade presencial não se preparam com antecedência para a realização de testes/provas. Esses últimos achados são alarmantes visto que o planejamento e a preparação para a realização de um objetivo influencia diretamente nos resultados da execução. Thibodeaux et al. (2016) apontam que estudantes que se prepararam e planejavam pouco tinham desempenho inferior a aqueles que planejam uma atividade. O pouco uso da estratégia E3 por estudantes da modalidade presencial pode ser explicado pelo uso do tempo em atividades remunerativas, visto que, conforme a Tabela 4 , é um público mais maduro e que geralmente possui famílias constituídas.

De forma geral, por meio da análise descritiva, cerca de $75 \%$ dos estudantes da modalidade a distância e $71 \%$ dos estudantes da modalidade presencial utilizam mais que $50 \%$ das estratégias encontradas em estudantes tidos como autorregulados. O percentual geral de uso das estratégias entre as duas modalidades não apresentou grandes discrepâncias, o que vai ao encontro dos achados de Sizoo et al. (2003), ao afirmarem que não existem diferenças significativas entre ambas as modalidades.

O segundo objetivo específico propôs analisar se a adoção das estratégias pode ser explicada a partir do gênero, idade e semestre em ambas as modalidades de ensino estudadas. Nessa análise foi aplicado o teste $t$ para amostras independentes utilizando o aprendizado autorregulado como variável independente. Para a realização dos testes as variáveis analisadas foram agregadas em dois grupos: gênero - masculino e feminino; idade até 30 anos e mais que 30 anos; semestre em curso - até o $4^{\circ}$ semestre e do $5^{\circ}$ ao $8^{\circ}$ semestre; e modalidade de ensino - presencial e EAD (Tabelas 5-7).

Tabela 5

\section{Testes de igualdade de médias para estágio no curso nas modalidades presencial e a distância}

\begin{tabular}{|c|c|c|c|c|c|c|c|c|c|}
\hline & \multirow[b]{2}{*}{$\mathrm{N}$} & \multirow[b]{2}{*}{ Média } & \multirow[b]{2}{*}{$\begin{array}{l}\text { Desvio } \\
\text { Padrão }\end{array}$} & \multirow[b]{2}{*}{$\begin{array}{c}\text { Erro } \\
\text { Padrão }\end{array}$} & \multicolumn{3}{|c|}{ Teste $t$} & \multicolumn{2}{|c|}{ Teste de Levene } \\
\hline & & & & & $t$ & $\begin{array}{l}\text { Grau de } \\
\text { liberdade }\end{array}$ & sig. & $\mathrm{F}$ & sig \\
\hline \multicolumn{10}{|l|}{ Semestre } \\
\hline \multicolumn{10}{|c|}{$\begin{array}{l}\text { Modalidade } \\
\text { à distância }\end{array}$} \\
\hline até 4 & 101 & 5,3970 & 0,82601 & 0,8219 & 0,960 & 169 & 0,339 & \multirow[t]{2}{*}{0,229} & \multirow[t]{2}{*}{0,633} \\
\hline 5 ao 8 & 70 & 5,2714 & 0,86362 & 0,10322 & 0,952 & 144,227 & 0,343 & & \\
\hline \multicolumn{10}{|c|}{$\begin{array}{c}\text { Modalidade } \\
\text { presencial }\end{array}$} \\
\hline até 4 & 37 & 5,5216 & 0,69046 & 0,11351 & 2,158 & 129 & 0,033 & \multirow[t]{2}{*}{5,478} & \multirow[t]{2}{*}{0,021} \\
\hline 5 ao 8 & 94 & 5,1277 & 1,02143 & 0,10535 & 2,544 & 96,901 & 0,013 & & \\
\hline
\end{tabular}

Nota. Fonte: Dados da pesquisa (2017).

O teste $\mathrm{F}$ na modalidade a distância foi igual a 0,229, com um nível de significância (sig.)de 0,633 e na modalidade presencial de 5,478, com significância de 0,021 (Tabela 7). Como o sig.> 0,05 aceita-se a hipótese nula $\mathrm{HO}$ de igualdade e supõe-se que as amostras tenham sido extraídas de população com a mesma variância para a modalidade a distância; enquanto na modalidade presencial aceita-se $\mathrm{H} 1$ de haver diferenças significativas entre as médias.

Na modalidade a distância não é possível perceber aumento ou redução no uso de estratégias autorreguladas e cursar o início ou fim do curso, pois o nível de significância foi acima de 5\%. Tais resultados indicam a ausência de incentivos/desenvolvimento de atitudes autorreguladas que promovam maior autonomia do aprendizado ao longo do curso de graduação na modalidade à distância. Esse achado converge com os resultados encontrados por Lima e Bruni (2012), ao indicar a impossibilidade de estabelecer relação entre as variáveis analisadas. Esses resultados levam refletir que, se o desenvolvimento de habilidades autorregulatórias reflete maior ou menor sucesso (Zimmerman \& Martinez-Pons 1986) e se não houver incentivos ao desenvolvimento dessas habilidades o estudante deixará o ensino superior com as mesmas habilidades com que ingressou na instituição, fato não desejável 
segundo órgãos internacionais do (AECC, 1990) e (AICPA, 2000) para o desenvolvimento do aprendizado permanente. Gonçalves e Vagula (2012) concordam que diante a explosão de fontes alternativas do conhecimento e ao pouco tempo em se trabalhar todas essas informações, o professor também deve fomentar o desenvolvimento de habilidades autônomas nos estudantes, como professor-mediador e garantir o aprendizado permanente. Dessa forma, torna-se necessário repensar o papel do professor em sala de aula de modo promover, também, o desenvolvimento de habilidade autorregulatórias em alunos de contabilidade e/ou de outras áreas.

Entretanto, quando analisados os testes de igualdade de média da modalidade presencial foi possível constatar que o semestre em que o estudante se encontra influencia o uso/apropriação de estratégias autorreguladas. A média para os estudantes até o quarto semestre em curso de contabilidade foi de 5,5216 (significância 3,3\%) enquanto aqueles no estágio do quinto ao oitavo semestre apresentaram média de 5,1277 (significância 1,3\%). Apesar de pouca diferença entre as médias percebe-se que estudantes da modalidade presencial até a metade do curso de graduação tendem a utilizar mais estratégias de autorregulação.

Tais achados permitem rejeitar a hipótese (H2) corroborada por Sizoo et al. (2003), que estabelece a apropriação semelhante de estratégia de aprendizado autorregulado entre as modalidades presencial e a distância quando analisado o estágio em curso; e aceitar a hipótese (H4) indicada por Lima e Bruni (2013) ao indicar ausência de relação entre a utilização de estratégia e o semestre em curso, com exceção do curso na modalidade presencial que houve maior utilização de estratégias em períodos iniciais.

A Tabela 6 apresenta o teste de igualdade de médias quando analisados os gêneros feminino e masculino e o uso de estratégias autorreguladas na modalidade presencial e a distância. Em ambas as modalidades de ensino não é possível estabelecer relação entre o uso das estratégias e o sexo dos estudantes de contabilidade (sig > 5\%). Esse resultado indica a rejeição da hipótese 1 (H1), corroborado pelos achados de Pavesi (2015), Lima e Bruni (2012) e Bembenutty (2007), que afirmam ser o sexo feminino aquele que mais se apropria de estratégias autorreguladas. Gonçalves e Vagula (2012) ao estudarem a modificabilidade cognitiva estrutural afirmam que o estímulo ao conhecimento de um novo objeto determina se o indivíduo possua mais ou menos estrutura de modificabilidade estrutural rígida. Dessa forma, é esperado que o gênero não seja fator de distinção de habilidades, mas sim os estímulos que foram desenvolvidos ao longo da vida do indivíduo.

Tabela 6

Testes de igualdade de médias para gênero nas modalidades presencial e a distância

\begin{tabular}{|c|c|c|c|c|c|c|c|c|c|}
\hline & \multirow[b]{2}{*}{$\mathbf{N}$} & \multirow[b]{2}{*}{ Média } & \multirow[b]{2}{*}{$\begin{array}{l}\text { Desvio } \\
\text { Padrão }\end{array}$} & \multirow[b]{2}{*}{$\begin{array}{c}\text { Erro } \\
\text { Padrão }\end{array}$} & \multicolumn{3}{|c|}{ Teste $t$} & \multicolumn{2}{|c|}{ Teste de Levene } \\
\hline & & & & & $t$ & $\begin{array}{l}\text { Grau de } \\
\text { liberdade }\end{array}$ & sig. & F & sig. \\
\hline \multicolumn{10}{|l|}{ Gênero } \\
\hline \multicolumn{10}{|c|}{$\begin{array}{l}\text { Modalidade a } \\
\text { distância }\end{array}$} \\
\hline Masculino & 77 & 5,2442 & 0,86686 & 0,09879 & $-1,432$ & 169 & 0,154 & \multirow{2}{*}{0,253} & \multirow{2}{*}{0,615} \\
\hline Feminino & 94 & 5,4287 & 0,81513 & 0,08407 & $-1,423$ & 158,159 & 0,157 & & \\
\hline \multicolumn{10}{|l|}{$\begin{array}{c}\text { Modalidade } \\
\text { presencial }\end{array}$} \\
\hline Masculino & 41 & 5,0805 & 0,98697 & 0,15414 & $-1,286$ & 129 & 0,201 & \multirow{2}{*}{0,045} & \multirow{2}{*}{0,833} \\
\hline Feminino & 90 & 5,3111 & 0,93526 & 0,09858 & $-1,260$ & 73,864 & 0,211 & & \\
\hline
\end{tabular}

Nota. Fonte: Dados da pesquisa (2017).

Por fim, o teste de igualdades de médias foi realizado para analisar se a idade influencia o maior ou menor uso de estratégias autorreguladas na modalidade presencial e a distância, conforme a Tabela 7. Na modalidade de ensino a distância não é possível perceber relação entre maior ou menor idade e uso/apropriação de estratégias autorreguladas, uma vez que o nível de significância foi maior que $5 \%$. 
Tabela 7

Testes de igualdade de médias para idade nas modalidades presencial e a distância

\begin{tabular}{|c|c|c|c|c|c|c|c|c|c|}
\hline & \multirow[b]{2}{*}{$\mathbf{N}$} & \multirow[b]{2}{*}{ Média } & \multirow[b]{2}{*}{$\begin{array}{l}\text { Desvio } \\
\text { Padrão }\end{array}$} & \multirow[b]{2}{*}{$\begin{array}{c}\text { Erro } \\
\text { Padrão }\end{array}$} & \multicolumn{3}{|c|}{ Teste t } & \multicolumn{2}{|c|}{$\begin{array}{l}\text { Teste de } \\
\text { Levene }\end{array}$} \\
\hline & & & & & $\mathbf{t}$ & $\begin{array}{l}\text { Grau de } \\
\text { liberdade }\end{array}$ & sig. & $\mathbf{F}$ & sig \\
\hline \multicolumn{10}{|c|}{ Idade } \\
\hline \multicolumn{10}{|c|}{$\begin{array}{l}\text { Modalidade a } \\
\text { distância }\end{array}$} \\
\hline até 30 & 97 & 5,3021 & 0,88117 & 0,89947 & $-0,774$ & 169 & 0,440 & & \\
\hline$>30$ & 74 & 5,4027 & 0,78844 & 0,09165 & $-0,786$ & 164,692 & 0,433 & 1,820 & 0,179 \\
\hline \multicolumn{10}{|c|}{$\begin{array}{c}\text { Modalidade } \\
\text { presencial }\end{array}$} \\
\hline até 30 & 75 & 5,0653 & 0,96443 & 0,11136 & $-2,457$ & 129 & 0,015 & & \\
\hline$>30$ & 56 & 5,4714 & 0,89640 & 0,11979 & $-2,483$ & 122,915 & 0,014 & 0,561 & 0,455 \\
\hline
\end{tabular}

Nota. Fonte: Dados da Pesquisa (2017).

Entretanto, quando analisados os discentes da modalidade de ensino presencial os resultados do teste de igualdade de média apontaram diferenças significativas entre a relação aprendizado autorreguladas e a idade dos alunos analisados. Dessa forma, discentes com idade superior a 30 anos apresentaram maior média $(5,4714$, significância de $1,5 \%)$, quando comparados com aqueles com idade inferior a 30 anos (5,0653, significância de 1,4\%). Esses achados convergem para os achados de Lima e Bruni (2012) e Mayville (2007), ao afirmarem que a idade influencia a apropriação de estratégias autorreguladas, portanto, se aceita a hipótese $3(\mathrm{H} 3)$. Esse achado pode ser explicado pelo maior desenvolvimento de habilidades autorregulatórias acumuladas por indivíduos com maior idade, características importantes ao conhecimento permanente.

Com o objetivo de verificar as grandezas associadas às variáveis modalidade de ensino (presencia ou a distância), semestre, idade e gênero, foi utilizada a Análise Fatorial (Tabela 8). A principal função das diferentes técnicas de análise fatorial é reduzir uma grande quantidade de variáveis observadas em um número menor de fatores (Figueiredo \& Silva Júnior, 2010).

Tabela 8

\section{Componentes principais}

\begin{tabular}{ccccccc}
\hline Componente & \multicolumn{3}{c}{ Valores próprios iniciais } & \multicolumn{2}{c}{$\begin{array}{c}\text { Somas de extração de carregamentos ao } \\
\text { quadrado }\end{array}$} \\
\cline { 2 - 6 } & Total & \% de variação & \% cumulativa & Total & $\begin{array}{c}\text { \% de } \\
\text { variação }\end{array}$ & \% cumulativa \\
$\mathbf{1}$ & $\mathbf{3 , 4 5 4}$ & $\mathbf{3 4 , 5 4 3}$ & $\mathbf{3 4 , 5 4 3}$ & $\mathbf{3 , 4 5 4}$ & $\mathbf{3 4 , 5 4 3}$ & $\mathbf{3 4 , 5 4 3}$ \\
$\mathbf{2}$ & $\mathbf{1 , 1 3 0}$ & $\mathbf{1 1 , 2 9 9}$ & $\mathbf{4 5 , 8 4 2}$ & $\mathbf{1 , 1 3 0}$ & $\mathbf{1 1 , 2 9 9}$ & $\mathbf{4 5 , 8 4 2}$ \\
$\mathbf{3}$ &, 902 & 9,019 & 54,861 & & & \\
$\mathbf{4}$ &, 851 & 8,510 & 63,371 & & \\
$\mathbf{5}$ &, 811 & 8,107 & 71,478 & & \\
$\mathbf{6}$ &, 724 & 7,237 & 78,714 & & \\
$\mathbf{7}$ &, 660 & 6,605 & 85,319 & & \\
$\mathbf{8}$ &, 580 & 5,805 & 91,123 & & \\
$\mathbf{9}$ &, 486 & 4,858 & 95,982 & & \\
$\mathbf{1 0}$ &, 402 & 4,018 & 100,000 & & \\
\hline
\end{tabular}

Nota. Fonte: Dados da pesquisa (2017).

A extração de fatores pelo critério Latent Root é o mais utilizado na análise fatorial e admite a utilização autovalor (eigen values) maior que um. Dessa forma, o primeiro 
componente consegue explicar $35,54 \%$ da variância total dessa modalidade enquanto o segundo componente explica apenas $11,30 \%$.

\section{Tabela 9}

\section{Matriz de componente rotativo com o método varimax}

\begin{tabular}{c|c|c|c|c|c|c|c|c|c|c|c}
\hline Componente & P1 & P2 & P3 & P4 & P5 & P6 & P7 & P8 & P9 & P10 \\
\hline 1 &, $\mathbf{7 1 2}$ & $\mathbf{7 3 5}$ & $\mathbf{5 7 9}$ &, $\mathbf{6 1 5}$ &, $\mathbf{4 7 6}$ &, $\mathbf{4 2 2}$ &,- 147 &, 292 &, 566 &, 617 \\
2 &,- 124 &, 054 &, 308 &, 220 &, 427 &, 343 & $\mathbf{, 7 9 1}$ & $\mathbf{, 6 3 2}$ &, 088 &, 340 \\
\hline
\end{tabular}

Nota. Fonte: Dados da pesquisa (2017).

Menezes (2006) aborda critérios de exclusão de cargas fatoriais com o objetivo de uma distribuição mais homogênea dos itens do modelo: valor absoluto da carga fatorial for menor < 0,32 ; cargas fatoriais similares em um mesmo fator serem menor que $0,1(<0,1)$ e um fator é formado por mais de um item.

De acordo com tal modelo foram excluídos os itens P5 (Tomada de apontamentos) e P6 (Estrutura ambiental) pelo fato dos valores serem menores que 0,1, quando comparados os dois componentes. A questão 7 também foi expurgada do estudo pela média das respostas estar abaixo do ponto médio (4). Não caracteriza uma estratégia adotada, além do nível de significância do coeficiente de Pearson estar maior que 0,05 em correlação às demais questões. Dessa forma, o primeiro componente (Tabela 9) está relacionado ao planejamento e controle das estratégias da autoaprendizagem utilizada pelos discentes e o segundo componente corresponde à utilização de técnicas de memorização como artifício de autoaprendizagem. A Tabela 10 apresenta a utilização dos fatores encontrados por meio da análise fatorial divididos pela modalidade de ensino.

Tabela 10

\section{Fatores de estratégias autorreguladas por modalidade de ensino}

\begin{tabular}{cc|c|c|c|c|c}
\hline \multicolumn{2}{c|}{ Modalidade de ensino do estudante } & $\mathbf{N}$ & Mínimo & Máximo & Média & Desvio padrão \\
\hline \multirow{2}{*}{ Distância } & Fator 1: planejamento e controle & 171 & 3,00 & 7,00 & 5,7710 &, 93620 \\
& Fator 2: Memorização & 171 & 1,00 & 7,00 & 4,7193 & 1,80282 \\
& N válido (de lista) & 171 & & & & \\
\hline \multirow{2}{*}{ Presencial } & Fator 1: planejamento e controle & 131 & 2,33 & 7,00 & 5,4504 & 1,03501 \\
& Fator 2: Memorização & 131 & 1,00 & 7,00 & 4,9160 & 1,73222 \\
& N válido (de lista) & 131 & & & & \\
\hline
\end{tabular}

Nota. Fonte: Dados da pesquisa (2017).

Verifica-se na Tabela 10 a existência dos fatores do planejamento e memorização, conforme indicados por meio da análise fatorial. Percebe-se que os discentes da modalidade a distância tendem a utilizar com maior frequência o fator de planejamento e controle, enquanto os discentes da modalidade presencial utilizam estratégias ligadas à memorização. Os testes de qualidade desses dados podem ser observados na Tabela 11. Esses achados podem refletir as características de cada modalidade de ensino. Para Niemi, Harju, Vivitsou, Viitanen, Multisilta e Kuokkanen (2014), os estudantes da modalidade a distância aprendem a administrar o tempo e a se tornar atores no processo de aprendizagem, enunciado que pode explicar a redução do fator a utilização da estratégia do planejamento e controle, essencial a modalidade de ensino em questão. Por outro lado, estudantes da modalidade presencial são submetidos constantemente à verificação de aprendizagem, geralmente sem auxílios de materiais de estudos, o que pode explicar a maior utilização de estratégias de memorização. 
Tabela 11

Qualidade dos dados na análise fatorial

\begin{tabular}{c|c|c}
\hline Dimensionalidade & Referência & Resultado \\
\hline \multirow{2}{*}{ Índice Kaiser-Meyer-Olkin (KMO) } & Maior que $0,7=$ & \multirow{2}{*}{0,820} \\
& Desejável & \\
\cline { 2 - 2 } & $\begin{array}{c}\text { Entre } 0,5 \text { e } 0,7= \\
\text { Aceitável }\end{array}$ & \\
\cline { 2 - 2 } & $\begin{array}{c}\text { Menor que } 0,5= \\
\text { Inaceitável }\end{array}$ & 0,000 \\
\hline Teste de esfericidade de Bartlett & $\begin{array}{c}\text { Nível de significância } \\
<0,05\end{array}$ & Resultado \\
\hline Confiabilidade & Referência & 0,762 \\
\hline Alfa de Cronbach & Maior que $0,60=$ & confiável \\
\hline
\end{tabular}

Nota. Fonte: Dados da pesquisa (2017).

Para análise da dimensionalidade, foi considerado o teste Kaiser-Meyer-Olkin, com intuito de verificar o índice de adequação da amostra. $O$ resultado encontrado foi de 0,820 , o que é considerado desejável, segundo Menezes (2006). Já outro teste referente a dimensionalidade utilizado foi o de esfericidade de Bartlett, para o qual o resultado do nível de significância foi menor que 0,05 (Tabela 11). De forma geral, foi identificado o perfil dos discentes autorregulados por meio da média das respostas, conforme Tabela 12.

Tabela 12

Perfil de um autorregulado através da média

\begin{tabular}{l|l|l|l|l|l}
\hline & $\mathbf{N}$ & Mínimo & Máximo & Média & Desvio padrão \\
\cline { 2 - 6 } Estratégias autorreguladas & 302 & 2,29 & 7,00 & 5,5137 &, 97026 \\
\hline
\end{tabular}

Nota. Fonte: Dados da pesquisa (2017).

A Tabela 12 indica que a média dos estudantes da modalidade presencial e a distância foi 5,5137 superior ao ponto médio de utilização das estratégias pelos discentes de contabilidade, dados que sugerem um alto nível de utilização de estratégias de autorregulação. Nota-se que o desvio padrão é baixo $(0,97026)$ demonstrando que os dados não são divergentes e com baixa dispersão. Trata-se de um achado importante, porém, enfatiza-se que o indivíduo tende a reagir às necessidades cognitivas exigidas (Gonçalves \& Vagula, 2012) de modo que, quanto mais habilidades autorreguladas forem trabalhadas ainda na graduação em contabilidade maior será o sucesso profissional e a preparação para lidar com a velocidade e a quantidade informativa.

\section{CONCLUSÃO}

À luz de quatorze estratégias de autorregulação da aprendizagem identificadas por Zimmerman e Martinez-Pons (1986), foi efetuada uma análise comparativa de uso de estratégias de aprendizado autorregulado em discentes graduandos em Ciências Contábeis na modalidade presencial e a distância em duas Instituições de Ensino Superior privadas, localizadas em Feira de Santana-BA (UNOPAR) e em Salvador-BA (FVC).

Buscou-se analisar as estratégias de autorregulação da aprendizagem adotadas por discentes de contabilidade na modalidade presencial e a distância e relacionar a adoção das estratégias autorreguladas ao gênero, idade e semestre nas respectivas modalidades.

De forma geral não foi possível perceber significativas diferenças entre as modalidades, quando analisadas as características de aprendizado autorregulado utilizadas pelos discentes de contabilidade, fato que preocupa, uma vez que é desejável (sobretudo) de estudantes da modalidade a distância maior autonomia para planejar, estruturar e organizar o seu aprendizado.

Aproximadamente $70 \%$ da amostra apontou usar mais que $50 \%$ das estratégias de aprendizado autorregulado em ambas as modalidades de ensino. Os resultados apontaram 
que a "autoconsequência" (E7) é a estratégia menos utilizada pelos estudantes de contabilidade, de forma a não existir "autopremiação" e/ou "autopunição" pela ocorrência de sucesso ou insucessos no desempenho de uma atividade. Na modalidade presencial, mais que a metade da amostra apontou baixa utilização da estratégia para o estabelecimento de objetivos e planejamento (E3) indicando que estudantes dessa modalidade não se preparam com antecedência para a realização de provas/exames. Tal comportamento pode influenciar nos resultados das avaliações realizadas, uma vez que é imprescindível a sistematização e a prática dos conteúdos a serem estudados em contabilidade e os estudos "em véspera" podem não ser suficientes e resultar em rendimentos mais baixos. Na modalidade a distância a amostra indicou utilizar mais que $70 \%$ das estratégias de aprendizado autorregulado, com exceção da estratégia "autoconsequência" (E7).

Os testes de igualdade de médias apontaram a impossibilidade de estabelecer relações para uso/apropriação de aprendizado autorregulado e semestre, gênero e idade para a modalidade a distância, enquanto na modalidade presencial estudantes até a metade do curso e aqueles com idade superior a 30 anos tendem a fazer mais uso de estratégias autorreguladas. A não apropriação de estratégias de autorregulação ao longo do curso pode ser um indício preocupante na educação continuada dos formandos em contabilidade, pois se trata de uma ciência que está em constante mudanças normativas, por exemplo.

A análise fatorial permitiu encontrar a presença de dois fatores: o de planejamento e o de memorização. Evidenciou-se que estudantes da modalidade a distância tendem a utilizar com maior intensidade o fator de planejamento e controle enquanto os estudantes da modalidade presencial as estratégias ligadas à memorização. Porém, em geral, estudantes de contabilidade apresentaram uma forte utilização de estratégias autorreguladas.

Os resultados encontrados neste estudo e associados a outras pesquisas (apresentados ao longo do artigo) sobre a autorregulação da aprendizagem, permitem convergir para a teoria de Zimmerman e Martinez-Pons (1986), que relaciona a potencial presença que habilidades autorregulatórias proporcionam na vida acadêmica de estudantes. Com essa constatação, é essencial promover estudos que indiquem como trabalhar o desenvolvimento de habilidades autorregulatórias em cursos de graduação em ciências contábeis. Também é interessante a realização de estudos que sondem como a utilização de estratégias de autorregulação tem influenciado no desempenho dos profissionais em contabilidade.

Este estudo apresenta algumas limitações quanto à amostra estudada ser composta apenas por duas Instituições de ensino privado localizadas na Bahia e analisa apenas as variáveis gênero, idade e semestre. Sugere-se, portanto, o desenvolvimento de outras pesquisas, envolvendo variáveis que possam explicar melhor a utilização dessas estratégias por estudantes de ciências contábeis, assim como ampliar a amostra.

\section{REFERÊNCIAS}

Accounting Education Change Commission. (1990). Objectives of education for accountants: Position statement number one. Issues in Accounting Education, 5(1), 307-312.

American Institute Of Certified Public Accountants. (2000). Core Competency Framework for Entry into the Accounting Profession.

Arias, A. V., Barca Lozano, A., Gonzalez Cabanach, R., \& Núñez Pérez, J. C. (1999). Las estrategias de aprendizaje revisión teórica y conceptual. Revista latinoamericana de Psicología, 31(3).

Barnard-Brak, L., Paton, V. O., \& Lan, W. Y. (2010). Profiles in self-regulated learning in the online learning environment. The International Review of Research in Open and Distributed Learning, 11(1), 61-80.

Bembenutty, H. (2007). Self-regulation of learning and academic delay of gratification: Gender and ethnic differences among college students. Journal of advanced academics, 18(4), 586-616. 
Bergamin, P. B., Ziska, S., Werlen, E., \& Siegenthaler, E. (2012). The relationship between flexible and self-regulated learning in open and distance universities. The International Review of Research in Open and Distributed Learning, 13(2), 101-123.

Boruchovitch, E. (2004). A auto-regulação da aprendizagem e a escolarização inicial. Aprendizagem: processos psicológicos e o contexto social na escola, 2, 55-88.

Cavanaugh, T., Lamkin, M. L., \& Hu, H. (2012). Using a Generalized Checklist to Improve Student Assignment Submission Times in an Online Course. Journal of Asynchronous Learning Networks, 16(4), 39-44.

Castel, A. D., Murayama, K., Friedman, M. C., McGillivray, S., \& Link, I. (2013). Selecting valuable information to remember: Age-related differences and similarities in selfregulated learning. Psychology and Aging, 28(1), 232.

Castro, J.X., Miranda, G.J., \& Leal, E.A. (2016). Estratégias de aprendizagem dos estudantes motivados. Advances in Scientific and Applied Accounting, 09(1), 080-097.

Castro, R. F. (2016). Autorregulação da aprendizagem no ensino superior a distância: o que dizem os estudantes?. Revista Brasileira de Ensino Superior, 2(2), 15-26.

Chen, S.Y., \& Paul, R. (2003). Editorial: individual differences in web-based instruction-an overview. British Journal of Educational Technology, 34(4), 85-92.

Costa, J. J. M. (2001). Autoregulação da aprendizagem: para uma caracterização multidimensional do desempenho acadêmico. Dissertação de Doutoramento em Psicologia, especialização em Psicologia da Motivação e da Personalidade, não publicada, Faculdade de Psicologia e de Ciência da Educação da Universidade de Coimbra, Coimbra, Portugal.

Dias, R.A., \& Leite, L.S. (2010). Educação à distância: da legislação ao pedagógico. (2a ed.). Petrópolis: Vozes.

Eysenck, M.W., \& Keane, M.T. (1994). Psicologia cognitiva: um manual introdutório. Porto Alegre: Artes Médicas.

Figueira, A. P. C. (1994). Em torno do rendimento escolar. Dissertação de Mestrado em Psicologia, não publicada, especialização em Psicologia Pedagógica, Faculdade de Psicologia e de Ciências da Educação da Universidade de Coimbra, Coimbra, Portugal.

Figueiredo, D. B., Filho, \& Silva Júnior, J. A. D. (2010). Visão além do alcance: uma introdução à análise fatorial. Opinião pública, 16(1), 160-185.

Flavell, J. H. (1976). Metacognitive aspects of problem solving. The nature of intelligence, 231235.

Frison, L. M. B., \& de Moraes, M. A. C. (2010). As práticas de monitoria como possibilitadoras dos processos de autorregulação das aprendizagens discentes. Poíesis Pedagógica, 8(2), 144-158.

Gatti, B. A. (2013). Implicações e perspectivas da pesquisa educacional no Brasil cotemporâneo. Cadernos de pesquisa, (113), 65-81.

Gil, A. C. (1991). Como elaborar projetos de pesquisa. São Paulo: Atlas.

Gonçalves, C. E. de S., \& Vagula, E. (2012). Modificabilidade Cognitiva Estrutural De Reuven Feuerstein: Uma Perspectiva Educacional Voltada Para O Desenvolvimento. 9a ANPED SUL. Recuperado de http://www.ucs.br/etc/conferencias/index.php/anpedsul/9anpedsul/ paper/view/1106

Korkmaz, O., \& Kaya, S. (2012). Adapting Online Self-Regulated Learning Scale into Turkish. Turkish Online Journal of Distance Education, 13(1), 52-67.

Jones, M. H., Alexander, J. M., \& Estell, D. B. (2010). Homophily among peer groups members' perceived self-regulated learning. The Journal of Experimental Education, 78(3), 378-394. 
Lakatos, E. M., \& Marconi, M. A. (2004). Metodologia cientifica. (4a ed. rev. e ampl.). São Paulo, Brasil.

Lefrançois, G.R. (2008). Teorias da aprendizagem. São Paulo: Cengage Learning, Brasil.

Lima, R. N., Filho, \& Bruni, A. L. (2012). Aprendizado Autorregulado em Contabilidade: Diagnóstico, Dimensões e Explicações. Anais do Encontro da Anpad (EnAnpad), Rio de Janeiro, RJ, Brasil, 36.

Lima, N., Filho, Lima, G. A. S. F. de, \& Bruni, A. L. (2015). Self-regulated learning in accounting: diagnosis, dimensions and explanations. BBR-Brazilian Business Review, 12(1).

Luca, M. M. M. De, Gomes, C. A. S., Corrêa, D. M. M. C., \& Domingos, S. R. M. (2011). Participação Feminina na Produção Científica em Contabilidade Publicada nos Anais dos Eventos Enanpad, Congresso USP de Controladoria e Contabilidade e Congresso Anpcont. Revista de Contabilidade e Organizações, 5(11), 145-164.

Lynch, R., \& Dembo, M. (2004). The relationship between self-regulation and online learning in a blended learning context. The International Review of Research in Open and Distributed Learning, 5(2).

Martin, A. J., \& Dowson, M. (2009). Interpersonal relationships, motivation, engagement, and achievement: Yields for theory, current issues, and educational practice. Review of educational research, 79(1), 327-365.

Mayville, K. L. (2007). Knowledge construction, self-regulation, and technology strategies used by experienced online nursing students to actively engage in online learning. Tese (Doutorado em Filosofia), Universidade Capella.

Melchior, M. C. (1994). Avaliação pedagógica: função e necessidade. Porto Alegre: Mercado Aberto.

Menezes, I. G. (2006). Escala de Intenções Comportamentais de Comprometimento Organizacional (Eicco): Concepção, Desenvolvimento, Validação E Padronização. Dissertação de mestrado, Universidade Federal da Bahia, Salvador, BA, Brasil.

Nascimento, M, \& Junqueira, E. (2012). Análise do perfil do aluno de ciências contábeis na modalidade a distância e do seu desempenho na disciplina de contabilidade introdutória. Anais do Encontro da Anpad (EnAnpad), Rio de Janeiro, RJ, Brasil, 29.

Niemi, H., Harju, V., Vivitsou, M., Viitanen, K., Multisilta, J., \& Kuokkanen, A. (2014). Digital storytelling for 21st-century skills in virtual learning environments. Creative Education, 5(9), 657.

Pavesi, M. A. (2015). Análise da aprendizagem autorregulada de alunos de cursos à distância em função das áreas de conhecimento, faixa etária e sexo. Dissertação de mestrado, Universidade Estadual de Londrina, Londrina, PR, Brasil.

Polydoro, S. A. J., \& Azzi, R. G. (2009). Autorregulação da aprendizagem na perspectiva da teoria sociocognitiva: introduzindo modelos de investigação e intervenção. Psicologia da Educação, (29), 75-94.

Ribeiro, C. (2003). Metacognição: um apoio ao processo de aprendizagem. Psicologia: reflexão e crítica, 16(1), 109-116.

Richter, T., \& Schmid, S. (2010). Epistemological beliefs and epistemic strategies in selfregulated learning. Metacognition and Learning, 5(1), 47-65.

Rosário, P. (1999). Variáveis Cognitivo-motivacionais na Aprendizagem: As "Abordagens ao Estudo" em alunos do Ensino Secundário. Tese (Doutorado em Educação), Universidade do Minho, Braga, Portugal.

Rosário, P., Nunes, T., Magalhães, C., Rodrigues, A., Pinto, R., \& Ferreira, P. (2010). Processos de auto-regulação da aprendizagem em alunos com insucesso no $1^{\circ}$ ano de Universidade. Psicologia Escolar e Educacional, 14(2), 349-358. 
Testa, M. G., \& Luciano, E. M. (2010). A influência da autorregulação dos recursos de aprendizagem na efetividade dos cursos desenvolvidos em ambientes virtuais de aprendizagem na internet. REAd-Revista Eletrônica de Administração, 16(2), 481-513.

Thibodeaux, J., Deutsch, A., Kitsantas, A., \& Winsler, A. (2016). First-Year College Students' Time Use. Journal of Advanced Academics, 28(1), 5-27.

Schleifer, L. L., \& Dull, R. B. (2009). Metacognition and performance in the accounting classroom. Issues in Accounting Education, 24(3), 339-367.

Schunk, D. H. (2001). Self-regulation through goal setting. ERIC Clearinghouse on Counseling and Student Service, University of North Carolina at Greensboro.

Simão, A. M. da V., \& Frison, L. M. B. (2013). Autorregulação da aprendizagem: abordagens teóricas e desafios para as práticas em contextos educativos. Cadernos de Educação, (45), 02-20.

Sizoo, S., Malhotra, N. K., \& Bearson, J. M. (2003). Preparing students for a distance learning environment: A comparison of learning strategies of in-class and distance learners. Journal of Educational Technology Systems, 31(3), 261-273.

Sternberg, R. J. (2013). Psicologia cognitiva (5a ed.). São Paulo: Cengage Learning.

Xu, M., Benson, S. N. K., Mudrey-Camino, R., \& Steiner, R. P. (2010). The relationship between parental involvement, self-regulated learning, and reading achievement of fifth graders: A path analysis using the ECLS-K database. Social Psychology of Education, 13(2), 237269.

Zimmerman, B. J., \& Martinez-Pons, M. (1986). Development of a structured interview for assessing student use of self-regulated learning strategies. American Educational Research Journal, 23, 614-628.

Zimmerman, B. J., Bonner, S., \& Kovach, R. (1986). Developing self-regulated learners; Beyond achievement to self-efficacy. Washington: American Psychological Association.

Zimmerman, B. J. (2000). Self-efficacy: an essential motive to learn. Contemporary Educational Psychology, 25(1), 82-91.

Zimmerman, B. J. (2001). Theories of self-regulated learning and academic achievement: An overview and analysis. NJ: Lawrence Erlbaum Associates, 25(1), p. 3-17.

Zimmerman, B. J. (2002). Becoming a self-regulated learner: An overview. Theory into practice, 41(2), 64-70. 